\title{
THE SUMMER MEETING IN EUGENE
}

The seventy-fourth summer meeting of the American Mathematical Society was held in Eugene, Oregon, from Tuesday through Friday, August 26-29, 1969. All sessions were held on the campus of the University of Oregon. There were 1398 persons in attendance, including 824 members of the Society. This meeting of the Society was held in conjunction with meetings of the Mathematical Association of America, the Society for Industrial and Applied Mathematics, $\mathrm{Pi} \mathrm{Mu}$ Epsilon, and $\mathrm{Mu}$ Alpha Theta.

There were two sets of Colloquium Lectures, each consisting of four lectures. Professor Raoul Bott of Harvard University presented the first series of Colloquium Lectures, entitled On the periodicity theorem of the classical groups and its applications. Professor Bott was introduced at his four lectures by Professors C. B. Morrey, Everett Pitcher, V. L. Shapiro, and R. S. Palais. The second set of Colloquium Lectures was given by Professor Harish-Chandra of the Institute for Advanced Study. His subject was Harmonic analysis on semisimple Lie groups. The four presiding officers at Professor Harish-Chandra's lectures were Professors C. B. Morrey, C. E. Rickart, Hyman Bass, and V.S. Varadarajan.

By invitation of the Committee to Select Hour Speakers for Annual and Summer Meetings, there were three invited addresses. Professor Robion C. Kirby of the University of California, Los Angeles, presented a lecture entitled $O n$ the existence and uniqueness of triangulations of manifolds. He was introduced by Professor P. Emery Thomas. Professor Murray Gertenhaber of the University of Pennsylvania lectured on Algebraic deformation theory. The introduction of Professor Gerstenhaber was made by Professor C. W. Curtis. The third invited speaker was Professor Paul F. Baum of Brown University. The title of his lecture was Vector fields and Gauss-Bonnett. Professor Baum was introduced by Professor Harley Flanders.

There were twenty-eight sessions for the presentation of contributed papers. The following persons presided at these sessions: P. M. Anselone, D. E. Arganbright, S. P. Avann, R. B. Barrar, R. A. Beaumont, F. J. Flaherty, J. B. Garnett, Myron Goldstein, R. W. Heath, D. P. Kraines, S. P. Lloyd, A. T. Lonseth, E. A. Maier, E. A. Michael, H. Y. Mochizuki, D. G. Moursund, Michael Papadopoulos, Father J. H. Raymond, P. L. Renz, K. A. Ross, B. M. Schreiber, W. P. Scott, A. L. Stone, J. M. Van Buskirk, L. E. Ward, Jr., V. C. Williams, C. R. B. Wright, F. H. Young. 
The Council met on August 26, 1969 at 5:00 P.M. in Room 101 of the Erb Memorial Union. There was an intermission for dinner, with adjournment at 11:00 P.M. until 3:00 P.M. the following day.

The Secretary announced that the following 277 persons had been elected to ordinary membership:

Alameddine, Ahmad P., Oregon State University;

Aldrich, Theodare M., Cleveland State University;

Amberg, Bernhard, University of Texas, Austin;

Ameen, Donald Louis, Lowell Technological Institute;

Anderson, Charles Lloyd, University of Southwestern Louisiana;

Araya, Pedro A., Coca Cola Export Corporation;

Aupetit, Bernard H., Laval University;

Avadhani, Tangirala Venkata, Andhra University;

Bach, Huguette L., Sacramento State College;

Baker, Charles R., University of North Carolina, Chapel Hill;

Ballard, Glen S., Union College;

Basu, Jyoti P., Texas Technological College;

Baumann, Horst Gunther, Ohio State University;

Beem, John K., University of Missouri;

Belfi, Victor Allen, Rice University, Texas;

Belina, Charles L., Michigan State University;

Berger, Neil E., University of Illinois, Chicago Circle;

Berry, Michael L., West Virginia Wesleyan College;

Blakemore, Carroll F., Mankato State College;

Blundon, William J., Memorial University of Newfoundland;

Bogar, Gary Allan, Montana State University;

Briggs, William J., University of Pennsylvania;

Brockhouse, James E., Western College;

Brown, Joseph G., University of Iowa;

Bueckner, Hans F., General Electric Company, New York;

Burchard, Hermann G., University of Wisconsin;

Byrd, Kenneth A., North Carolina State University, Raleigh;

Caldwell, Christopher S., University of Maryland;

Callas, Nicholas P., Air Force Office of Scientific Research;

Carney, Joseph Dennis, West Virginia State College;

Carter, Joan C., University of Hawaii;

Chiu, William M., College of the School of the Ozarks;

Christensen, Koester L., 1209 Lake Shore Drive, Escanaba, Michigan;

Church, J. D., University of Kansas;

Ciment, Melvyn, New York University, Bronx;

Clarke, Thomas G., Cleveland State College;

Clifford, Jerrold R., State University of New York, Stony Brook;

Coggins, George F., Portland State College;

Cohen, Joel S., University of Maryland;

Cohen, Moses Elias, Michigan Technological University;

Collins, W. Robert, University of Massachusetts;

Connolly, Mary V., Villanova University;

Conrad, D., 55 Pierrepont Street, Brooklyn, New York;

Cook, Ted K., University of Cincinnati; 
Copley, John R., Kansas Wesleyan University;

Cowan, Jack D., University of Chicago;

Crone, Lawrence J., Clarkson College of Technology;

Crowley, Edward C., Northeastern University;

Csima, I., McMaster University;

Cullen, Thoedore J., California Polytechnical Institute;

Curley, William A., Stevens Institute of Technology;

Dankert, Gabriele, University of Waterloo;

Davis, Chester L., Western Kentucky University;

Davis, Joel, Oregon State University;

Deans, Christine H., 10803 N. Oregon Circle, Tampa, Florida;

Deconge, Sister M.S., Loyola University;

Derosmay, Joel, French Embassy, Brookline, Massachusetts;

Dykes, Donald Campbell, Kent State University;

Erdelsky, P. J., California Institute of Technology;

Erickson, Theodore S., University of Massachusetts;

Etheridge, William L., North Dakota State University;

Evans, Melvis T., Albany State College;

Fair, Wyman G., Midwest Research Institute;

Faltings, Kai, University of Texas, Austin;

Fary, Istvan, University of California, Berkeley;

Feigenbaum, S., University of Bridgeport;

Feingold, David, Electricite de France, Clamart, France;

Fischer, Michael John, Carnegie Mellon University;

Fitzpatrick, Philip M., Auburn University;

Fleming, Mark C., Ohio Technical College;

Flynn, Sister M. Norbert, Dominican College of Blauvelt;

Forsythe, Odene, Kentucky Wesleyan College;

Frampton, Robert V., California State Polytechnic College;

Francis, Eugene A., University of Puerto Rico;

Fraser, Donald A. S., University of Toronto;

Frey, Laurence, New York College, Board of Education, Brooklyn, New York;

Friedmann, William E., Syracuse University;

Frye, Wyndham M., Tusculum College;

Gabriel, John R., Argonne National Laboratory;

Garb, Regina H., Newark State College;

Garcia, Myria A., University of Puerto Rico;

Getchell, Charles L., Lycoming College;

Gibons, Norman, Civic Center, City of Chicago;

Giroux, Andre, University of Montreal;

Glanze, Walter D., Bantam Books, Incorporated;

Glover, Harry C., University of Washington;

Gordon, Stanley L., Texas Instruments, Incorporated;

Goss, George K., Wesleyan University;

Grabowski, Sister M. Bonaventure, Felician College;

Grannick, Robert N., University of Chicago;

Grayston, Alan M., Perkin Elmer, Connecticut;

Grecos, Alkis, University of Texas, Austin;

Griffith, John S., Lakehead University;

Gruber, Gary R., Cambridge University Press; 
Guay, Merle D., University of New Hampshire;

Haggerty, Gerald B., University of Rhode Island;

Hall, William Spencer, University of Pittsburgh;

Harper, Robert E., West Virginia University;

Hayashi, Elmer K., University of Illinois, Urbana;

Hayes, Staley E., University of Texas, Austin;

Heard, Ann C., Kentucky State College;

Hearsey, Bryan V., University of Florida;

Heller, Max N., Juniata College;

Hennessey, John C., Loyola College;

Henry, Samuel S., University of South Carolina;

Herman, Michel, Centre de Mathematique de L'école Polytechnique, Paris, France;

Herrick, Daniel Lance, Barrington College;

Hethcote, Herbert Wayne, University of Iowa;

Hirschhorn, Harry J., Wright College;

Hirshon, Ronald, Polytechnic Institute of Brooklyn;

Holbrook, John Arthur, University of California, San Diego;

Holmkahl, Gunnar K. E., Asea Vasteras, Sweden;

Hoomani, J., University of Alabama, Huntsville;

Johnson, Nancy J., Moorhead State College;

Johnson, Ralph E., Duke University;

Jones, J. N., Chicago State College;

Joshi, Aravind K., University of Pennsylvania;

Julin, David T., Boston State College;

Kabak, Bertram S., Bronx Community College, City University of New York;

Keast, Patrick, University of Saint Andrews, Scotland;

Kelley, M. Elaine, University of Miami;

Khatchatooriantz, Ludwig A., University of California, San Diego;

Kilgore, Frank J., Catawba College;

Kimberling, Clark H., Northwest Missouri State College;

Kimble, Kenneth R., Ohio State University;

Koelzer, John G., Wartburg College;

Kohler, Alfred R., Long Island University;

Koller, Herbert, University of Bridgeport;

Ko, Youngso, Taejoin Presbyterian College, Korea;

Laetsch, Theodore, Illinois State University;

Lando, Barbara A., 774 Bevier Road, Piscataway Road, New Jersey;

Latil, Karen, University of Michigan;

Lau, Richard L., California Institute of Technology;

Leiper, Albert C., Sikorys Aircraft;

Lenihan, William James, University of Saskatchewan, Regina;

Leung, Dominic S. P., University of California, Berkeley;

Levan, Marijo O., South Eastern Missouri State College;

Lewis, John G., Saint Olaf College;

Lieb, Elliott H., Massachusetts Institute of Technology;

Lieb, Murry, Newark College of Engineering;

Lin, Tung Po, San Fernando State College;

Lipow, Carla, University of Wisconsin;

Liu, Mu Chou, University of Illinois;

Livesay, George Roger, Cornell University; 
Ly, Meng Kheng, Royale University, Dekg, Cambodia;

Lynde, Lowell F., Jr., Jonesboro Hodge High School;

MacDonald, Kenneth A., California State Polytechnic College;

MacHenry, Trueman, York University;

Manley, Paul L., University of Windsor, Ontario;

Marathe, Kishore B., University of Rochester;

Marques, R. Murillo, University of Campinas, Sao Paulo, Brazil;

Marrach, Nazem, Pan American College;

Marsden, Edwin L., Jr., Kansas State University;

Massey, Frank Jones, III, University of California, Berkeley;

McArthur, William G., Shippensburg State College;

McCann, Roger C., California State College, Los Angeles;

McConnell, Thomas, City University of New York;

McKibben, John, University of Massachusetts;

McMillan, Daniel R., Alabama College;

McMullen, P., Western Washington State College;

McNaughton, Robert, Rensselaer Polytechnic Institute;

Menmenon, Korandattil V., Dalhousie University;

Miller, Myron W., 12900 E. Cofax Street, Aurora, Colorado;

Mislin, Guido, Cornell University;

Montador, Robert Bruce, University of Montreal;

Morris, Carl W., Nassau Community College;

Moussa, Camara, Lycea Technique, Conakry, Guinea;

Murphy, David W., State University of New York, New Paltz;

Mushenheim, Harold, University of Dayton;

Nair, Vatuvaihedath C., Regional College of Education, Ajmer, India;

Nathan, Ravindra, Boeing Company;

Nel, Louis D., Carleton University;

Nicholson, Victor A., Kent State University;

Noelle, Gerald L., University of South Alabama;

Norris, Eugene Michael, University of Florida;

Oligner, Joseph E., National Center for Atmospheric Research;

O'Malley, Sister Mary Therese, Rosary College;

Orgas, Paul P., City University of New York;

Orgass, Richard J., IBM, Yorktown Heights, New York;

O'Sullivan, John M., State University of New York, Binghamton;

Oswald, K., Devry Institute of Technology;

Palmore, Julian Ivanhoe, III, University of California, Berkeley;

Pao, C. V., University of Pittsburgh;

Papp, Zoltan, Western Union Telegraph Company;

Peace, Karl E., Georgia Southern College;

Piper, W. Stephen, Purdue University;

Plummer, Robert, Bryn Mar College;

Powers, Kathryn A., Western Illinois University;

Powers, William H., Chrysler Corporation;

Prentiss, Donald K., McGraw-Hill Book Company;

Price, John F., Australian National University;

Price, Kenneth H., University of Texas, Austin;

Proschan, Joshua H., New York University;

Puri, Kewal Krishan, New York University, Bronx; 
Rabinowitz, Martin Myron, Bell Telephone Laboratories;

Rachman, Andrew B., St. Louis University;

Ramsay, R. T., North Carolina State University;

Ramsey, O. C., University of Iowa;

Reeder, Charles, Spring Hill College;

Reekie, G. Michael, Western Kentucky University;

Rees, Elmer, The University, Hull, England;

Riddell, Ronald C., University of British Columbia;

Rintel, Lionel, College of William and Mary;

Robbins, E. S., Plymouth State College;

Roberts, George T., Caldwell College;

Robinson, Raymond G., Jr., E. I. Dupont de Nemours and Company;

Rogers, Wanda J., Fresno State College;

Rorres, Chris, Drexel Institute of Technology;

Rose, Kermit L., Florida State University;

Roth, Walter J., University of North Carolina, Charlotte;

Russak, Ben, American Elsevier Publishing Company;

Ryan, Peter M., Dartmouth College;

Sahtout, M. M., Texas A and I University;

Sather, Duane Paul, University of Wisconsin;

Schaffer, Juan Jorge, Carnegie Mellon University;

Schoen, Alan H., NASA Electronics Research Center, Cambridge, Massachusetts;

Schultz, Norman L., John C. Calhoun State Junior College;

Schumann, Walter, École Polytechnique Federale, Switzerland;

Scott, Frank L., Ohio State University;

Scoville, Richard, Duke University;

Shachmut, William C., Grumann Aircraft Engineering Company;

Shapiro, Abraham W., UNIVAC, Philadelphia;

Shapiro, Joel Harold, University of Michigan;

Shapiro, Marcy D., Rutgers University;

Shaver, Linda D., Purdue University;

Shube, Beatrice, John Wiley and Sons;

Singh, Shri Krishna, Karnatak University;

Slemrod, Marshall, Brown University;

Smith, James R., Appalachian State University;

Sohn, Mun Gu, National University, Korea;

Sopka, John R., University of California, New Mexico;

Southworth, Raymond W., College of William and Mary;

Sox, Jason Loy, Jr., North Carolina State University;

Spencer, Thomas, Trenton State College;

Sribhibhadh, Siripongse, Prince of Songkla University, Bangkok, Thailand;

Stallard, Frank W., Georgia Institute of Technology;

Stefanini, Edward J., Clark University,

Steigerwalt, M. Susan, Pennsylvania State University;

Steinberg, Leon, Temple University;

Stoops, Glenn A., Litton Systems, Mellonics Division;

Storrer, Hans Heiner, McGill University, Montreal, Canada;

Strandberg, Richard M., University of Missouri, Rolla;

Suber, Harry H., Clemson University;

Sullivan, Wayne Garrett, Georgia Institute of Technology; 
Sweeney, James W., IBM, Rochester, Minnesota;

Swick, Kenneth E., Occidental College;

Tague, Jean M., Memorial University;

Tepper, David E., Temple University;

Thiam, Magatte, University of Dakar, Dakar, Senegal;

Thieleker, Ernest A., Argonne National Laboratory, Illinois;

Thornburg, James L., University of Missouri;

Tilley, John L., Mississippi State University;

Tollefson, Jeffrey L., Tulane University;

Torbert, Edgar C., III, Alabama College;

Torres, Euclides, Bishop College;

Tracy, Derrick Shannon, University of Windsor;

Treves, François, Purdue University;

Tsamisis, John C., Hercules, Incorporated;

Tucker, John, University of Waterloo;

Turner, Walter William, Western Michigan University;

Turner, Edward C., University of California, Los Angeles;

Ucci, Jack, Indiana University;

Vasilach, Serge, University Laval;

Viloria, Luis S., University of Zulia, Maracaibo, Venezuela;

Viswanathan, B., University of New Brunswick;

Vitale, Richard A., Brown University;

Wadkins, James R. J., Education Testing Service, Princeton, New Jersey;

Wakfer, Paul, University of Toronto;

Wall, Curtiss E., Olivet College;

Watkins, Kirby D., University of Texas, Arlington;

Weintraub, Sol, Queens College, City University of New York;

White, Arthur Thomas, II, Michigan State University;

Wiegman, Geraard J., Wolf Research and Development, Greenbelt, Maryland;

Wiltrakis, Edward J., North Carolina Wesleyan College;

Woodward, Melvin R., Indiana University of Pennsylvania;

Wong, Teddy T., RCA Institute;

Yakowitz, Sidney J., University of Arizona;

Yos, Ham, Royal University of Battambaug, Cambodia;

Zaret, Robert Bernard, Sperry Gyroscope, Long Island, New York;

Zippin, M., University of California, Berkeley.

The following individuals were elected to membership as nominees of the respective institutional members:

University of Alabama: Chao W. Chen, William T. Trotter, Jr.

Arizona State University: Henrik Bresinsky, Robert W. Fitzgerald.

University of Arizona: John A. Narvarte, S. Eugene Sims, Robert P. Smith.

Austin College: Wayne Charles Bell.

University of Bridgeport: Robert M. Lynott, John K. Marshall.

Brown University: John J. Anton, Robert L. Baker, Jr., Yeheskel Bar Ness, Chi Cheng Chen, David F. Fraser, Anthony A. Ganz, Stanley I. Grossman, Lorne S. Halabisky, Ulrich G. Haussmann, Donald E. McClure, Steven L. Shrier, Neil Stahl, Dennis George Weis, Stuart H. Zamlong.

California State College, Hayward: Kathleen E. Harding, Lie Yueh Lie, Michael Sze. 
California State College at Dominguez Hills: Gordon Matthews.

University of California, Berkeley: Charles F. Amelin, Frederick Backer, Jr., Elliott I. Bailis, Martin Bendersky, Jerome J. Blair, Richard Charles Blankenhorn, Robert E. Bowen, James R. Bunch, Charles J. Burke, Theodare Chang, Daniel S. Drucker, Samuel E. Ebenstein, Richard Steven Elman, Charles Fenimore, Kenneth L. Fields, Peter M. Goorjian, Gregory A. Hively, Chester Alan Jacewitz, Frederick W. Keene, Arthur J. Krener, Joseph G. Kupka, Hon Fai Lai, Charles F. Martin, Sheldon E. Newhouse, Roy Hiroshi Ogawa, Ole Osterby, Joseph F. Plante, R. Clark Robinson, Erik Rosenthal, Edwardine M. Schmid, Philip D. Straffin, Jr., Michael E. Taylor, Bret R. Tilson, Ignacio Unsain, William W. Wadge.

University of California, Davis: Gerald L. Geissert, Richard William Rose, Robert E. Tarone.

University of California, Los Angeles: Gerald A. Beer, Chang Shing Chen, Robert D. Crosby, Richard E. Healey, Jonathan E. Leech, Shigenori J. Morizumi, Dwight W. Read, Uwe K. Scholz, Jeanne C. Slaninger.

University of California, San Diego: Peter Georg Preuss, Raymond Joseph Zavodnik.

University of California, Santa Barbara: Hasan A. Celik, Bahattin Cengiz, Harry Melvin Freidman, Charles M. Joiner, Robert Keston, Sa Ge Lee, W. Keith Nicholson, Daniel John Randike, D. James Samuelson, Uppadahayala Venkata Satyanarayana, Gordon Whitnall, Elizabeth Rau Wilson.

Catholic University of America: Thomas F. Devlin, Hermann Dinges, Richard Alan Oberle, Sister H. Ambrosia Pohanka.

Central Michigan University: James K. Bidwell, Robert G. Clason.

Chicago State College: Peter Paul Pekar, Jr., Bernadette H. Perham, Dale J. Underwood.

University of Chicago: David L. Arp, James Francis G. Auchmuty, David C. Brown, Bruce D. Calvert, Ahmad Chalabi, Leslie Cohn, Antonio Conde, Fred J. Connell, Thomas K. Donaldson, Eduardo J. Dubuc, Clifton E. Ealy, Jr., David Eisenbud, Abiabdollah Fattahi, Pamela A. Ferguson, David Finkel, Stephen D. Franklin, William Francis Griffeth, Jr., John D. Hamilton, Ross M. Hamsher, James L. Heitsch, Domingo A. Herrero, Carlos Augusto S. Isnard, Martin L. Karel, Stanley O. Kochman, Hilton V. Machado, Anne R. MacWilliams, Ib H. Madsen, Stephen J. McAdam, M. Susan Montgomery, David T. Price, George W. Rusell, Lary Schiefelbusch, Harold W. Schneider, Claude L. Schochet, Raymond T. Shepherd, Martha K. Smith, Stephen B. Sontz, William H. Specht, Thomas Walsh, Stanley A. Weiss, Raphael Zahler.

University of Cincinnati: Joseph N. Lockhart, Carl P. Singer, Richard E. Strenk.

Brooklyn College, City University of New York: Allan M. Kaufman.

City College, City University of New York: Michael Doob, Robert Allen Katz, Martin Peres.

Herbert H. Lehman College, City University of New York: Shih Kuo Chow, Keitha Ann Landy, Linda G. Schiffman.

Hunter College, City University of New York: Linnie C. Cheng, Charles Gruber, Myra Jean Prelle.

Queens College, City University of New York: Lucy A. Santaniello.

Clemson University: James L. Crandall, Billy J. Holmes, George W. Marrah.

Cleveland State University: Walter R. Van Voorhis.

University of Colorado: James P. Coughlin.

Columbia University: Paul W. Anderson, Peter A. Larsen, Gerald V. Rowell. 
Connecticut College: Sarah J. Dunn.

Dalhousie University: P. P. Narayanaswani, Panayiotis A. Pittas.

Denison University: Michael M. Obletz, Michael A. Rotenberg, Clair E. Wuichet.

DePaul University: Anthony R. D’Angelo, William Edward Palluck, Andrew Postlewaite.

Eastern New Mexico University: Leroy M. Damewood, Dwight E. Lippe, Lyle E. Ochs.

East Texas State University: Dale R. Bedgood.

Emory University: Washington H. Carlisle, Etta Z. Falconer, Charles C. Lindner.

Florida Presbyterian College: C. Edward Reid, David L. Wilt.

Florida State University: Brenda J. Brannon, J. A. Conte, Wyatt G. Cooper, Paul L. Davis, James E. Desmond, Douglas J. Jones, Sun Sun Lee, Alice Maris, Wendell L. Motter, Paul A. Ohme, Thomas G. Parker, Walter C. Pine, Charles A. Prins, Jimmie A. Robertson, Brian J. Smith, Roger L. Taylor, Paul C. Woods.

University of Hawaii: Paul M. Perdew, Timothy R. Wilson.

Humboldt State College: Charles M. Biles, Russell J. Munsell, Roy W. Ryden.

Illinois State University: Thor Ronald Benson, Vincent Arthur Owles, James Lawrence Trudeau.

Iowa State University: George O. Strawn.

University of Manitoba: R. Padmanabhan, Barry Wolk.

University of Maryland: John David Anderson, William James Dahl, Dennis F. Deriggi, Marion Lee Edwards, Martin Frederick Gakenheimer, Katherine S. Goldman, Anita M. Goldner, James Klein, Kenneth C. Louden, James W. Nooman, Steven B. Oliphant, Dennis Phillips, Norman Purznsky, Mark Rockman, John A. W. Upton, Gary L. Van Velsir.

Memorial University of Newfoundland: Clayton W. Halfyard, Wayne M. Rowe, Wayne C. Russell.

Michigan State University: David W. Bash, Thomas R. Butis, J. Scott Downing, Richard A. Gibbs, Joseph T. Matti, James Lee Murphy, Daniel A. Nussbaum, William A. Simpson, Rolland L. Swank, S. Christelle Theusch.

University of Miami: Jeffry B. Fuqua, David M. Morris, Robert L. Snider, R. N. Soral.

University of Minnesota: Richard B. Amundson, Samuel Floyd Barger, Julio Esteban Bouillet, Alan David Boxer, Milton D. Brue, Louis B. Bushard, James Franklin Dillon, Norberto A. Fava, Orvald B. Haugsby, Ralph J. Herbert, Horacio Hernandez, David A. Judd, Wolodymyr R. Madych, James Milton Marver, Selmer Orlean Moen, Wayne R. Park, Won Joon Park, Ronald Edgar Reitz, Camilo Schmidt, Dieter Sam Schmidt, Joseph R. Schumi, John B. Stever, Daniel M. Sunday, Jr., Peter Michael Tomsich.

University of Mississippi: P. Keith Alford, Lester W. Jones, Jimmy Nanney.

University of Nebraska, Omaha: James B. Harrison, Jeffrey R. Kroll, Suzanne R. Neu.

New York University: John G. Aiken, Gray Jennings.

Northeastern University: Horst Behncke, Nurit Binenbaum, Alfredo A. Careaga, Li Chieh Chen, Chin Hung Chzng, Roger M. Firestone, Kenneth P. Goldberg, Stanley S. Goldberg, Erhard Gottschling, Samuel M. Graff, R. Douglas Gregory, Edward H. Grossman, Ole Hansen Hald, Matthew Halfant, Miriam Hausman, Paul Richard Levitt, Kyuya Masuda, Arthur Menikoff, Carlos Julio Moreno, Charles Vosco Rolli, Jay Rothman, Dennis Spellman.

Northern Illinois University: James Joyce, Stan Klasa, Axel Tobias Schreiner. 
Ohio Wesleyan University: Benjamin Lewis Barnes, Susan K. Woerner.

Oklahoma State University: James E. Dowdy, Joe D. Flowers, Roy M. Rakestraw. University of Oklahoma: Benigno B. Jorque, Churl S. Kim.

Old Dominion College: Samuel Franklin Hatton, Jr., Ted A. Holden, Stephen R. Pixley.

Oregon State University: Charles C. Burch, Jr., Wen Ning Hsieh, Ronald N. Knoshaug, Arthur E. Olson, Jr., James L. Southam, John W. Vander Beek, Lynn Taylor Winter.

Pennsylvania State University: Janet E. Ault, Gerard A. Bottorff, Willard G. Connor, David W. Crotty, James A. Earles, Charles F. Fennemore, Joseph C. Griffin, Carl B. Hurd, John P. Jones, Dale Z. Kilhefner, Margaret F. Kothmann, Jane C. Malbrock, Thomas L. Pirnot, Constantin Ragazas, Alan L. Selman, Douglas D. Smith, Chie Yunn Yu.

Pomona College: Brian M. Scott.

University of Puerto Rico: Gordon L. Gregg, Robert J. Lopez, Ralph L. Weatherwax.

Rensselaer Polytechnic Institute: William B. Day, Jonathan K. Millen, Arthur L. Price.

Rockefeller University: Pierre L. Van Moerbeke.

College of Saint Marie: C. C. Dube, F. P. Gagnon, Claude Gaulin.

Saint Mary's College of Maryland: Abigail A. Salyers.

University of Santa Clara: Charles F. Swart.

Texas A. \& I. University: Genaro Gonzalez.

University of Toledo: Jerry R. Beehler.

University of Toronto: John R. Baxter, Thomas Edward Cousins, A. M. Dawes, Kenneth A. Dunn, Chris J. Graddison, T. Michael Kavanagh, Ronald A. Kerman, Louisa Suk Wah Lam, R. J. Mackay, Michael J. Nelson, Ross L. Prentice, Joseph John Williams, James S. Witol.

Union College: Douglas Hatch, Dean Hoffman, Anthony R. Susko.

University of Virginia: Lavon Barry Page.

Virginia Commonwealth University: William L. Hoard, Kenneth V. Schmidt, R. Alvin Vaughn.

University of Waterloo: John Bailliend, Richard N. Burns, C. Haff, I. Pamela McWhirter, Donald W. Trim, Beverly Wilkinson, Thomas C. Wilson.

Wayne State University: Linda W. Brinn, Milton Chaika.

West Georgia College: Brenda G. Craven, Joseph Diestel, Edith H. Maxwell.

Western Michigan University: Wayne Bishop, Maurice L. Eggen, David B. Greenman.

Williams College: Ralph A. Gerra, Jr.

Winthrop College: Edward P. Guettler.

Wisconsin State University, Eau Claire: David R. Lund, Lawrence F. Wahlstrom, Marshall E. Wick.

It was announced that the following persons were admitted to membership in accordance with reciprocity agreements with the various mathematical organizations named:

Australian Mathematical Society: Richard J. Loy, Ahmad Shafaat, Alfred J. Van Der Poorten.

Osterreichische Mathematische Gesellschaft: Wliilbald Doerfler, Johann H. Hejtmanek, Fritz Schweiger. 
Societe Mathematique de Belgique: Roger Holvoet.

Societe Mathematique de France: Albert Badrikian, Jacques Couot, Jean Pierre

Gossez, Andre Kraganorr, Pierre Yves Leduc, Monique L. Pavel, Roger Temam.

Dansk Matematisk Forening: E. Jorgensen.

Deutsche Mathematiker Vereinigung: Friedrich Wille, Rudolf Wille.

The London Mathematical Society: Irving John Good, Patrick D. F. Ion, John David Knowles, Erwin H. Kronheimer, George O. Okikiolu, B. Rotman.

Indian Mathematical Society: Krishan Lal Duggal, Jagdish Saran Gupta, Chandra Mohan Joshi, P. N. Rathie, A. R. Reddy, J. P. Singhal.

Israel Mathematical Union: J. Schonheim, Uri Srebro.

Unione Matematica Italiana: Mario Miranda.

Mathematical Society of Japan: Mitsuo Fukushima, Satoru Igari, Makoto Ishida, Hiroyasu Ishimoto, Haruo Sunouchi.

Svenska Matematikersamfundet: Bernt Lindstrom.

Wiskundig Genoctschap te Amsterdam: G. C. L. Brummer, Jan D. Stegeman.

The institutions named below were elected to institutional membership:

California State Polytechnic College, Kellogg-Voorhis Campus, Pomona, California Chicago State College, Chicago, Illinois

Clarion State College, Clarion, Pennsylvania

Clark University, Worcester, Massachusetts

Cleveland State University, Cleveland, Ohio

Fairleigh Dickinson University, Teaneck, New Jersey

Lakehead University, Port Arthur, Canada

North Dakota State University, Fargo, North Dakota

St. Francis College, Loretto, Pennsylvania

Susquehanna University, Selinsgrove, Pennsylvania

University of Louisville, Louisville, Kentucky

University of New Brunswick, Fredericton, Canada

University of Winnipeg, Winnipeg, Canada

Virginia Commonwealth University, Richmond, Virginia

The following committees have been established and the following Presidential appointments have been made:

Committee on the By-laws and the Business Meeting, R. H. Bing, J. W. Green, E. Pitcher.

Committee on the Society View of Appropriate Conference Board Activity: S. MacLane, M. Protter, Chairman, L. Zippin.

Committee on National Awards and Public Representation: P. Cohen, N. Levinson, O. Zariski, Chairman.

Committee on Steele Prizes: F. Browder, S. S. Chern, M. Gerstenhaber, Chairman, E. Hewitt, N. Jacobson, S. Lang, J. Milnor, A. Seidenberg.

Organizing Committee for the Eugene Meeting: R. S. Freeman, replacing Larry $\mathrm{C}$. Grove, who resigned because of changes in his professional plans. This is a Joint Committee with the Mathematical Association of America. 
Organizing Committee for the Miami Meeting: additional members are H. L. Alder, ex officio, A. T. Butson, Mrs. Georgia Del Franco, H. T. Fleddermann, O. G. Harrold, Jr., ex officio, B. E. Howard, Sister Paul James Villemure, R. L. Kelley, R. D. Mazzagatti, Mrs. Agnes Rickey, G. L. Walker, ex officio. This is a Joint Committee with the MAA, of which the Chairman, already announced, is J. H. Curtiss. [The Secretary notes that the meeting was subsequently relocated after the Committee had done all the preliminary planning.]

Committee to Select Hour Speakers for Western Sectional Meetings: R. C. Lyndon for a full term beginning in 1970.

Tellers for the Referendum: Albert Wilansky, Murray Schechter.

Temporary observer for the National Computer Year of the Association for Computing Machinery: A. H. Taub.

Advisory Committee to the National Register: R. Wade Cole.

The following persons represented the Society at various functions as noted:

Arthur L. Hilt at the inauguration of Frederick Palmer Sample as President of Lebanon Valley College

Daniel Chang at the inauguration of Lawrence Park as President of Mansfield State College

Edward O. Nelson at the inauguration of Laurel D. Loftsgard as President of North Dakota State University

Phyllis A. Fox at the inauguration of James Karge Olsen as President of Paterson State College

E. F. Beckenbach at the inauguration of Harold Brown as President of the California Institute of Technology

Neal H. McCoy at the inauguration of David B. Truman as President of Mt. Holyoke College

E. H. Spanier on the CBMS Committee on Proposed Summer Research Institute.

The Council approved a Committee recommendation that the Gibbs Lecturer at the Winter meeting of 1970 be Professor Walter H. Munk from the Scripps Institute of Oceanography.

The Editorial Committees of the Proceedings, the Transactions and Mathematics of Computation all require an abstract, a descriptive title, AMS subject classification numbers, and key words and phrases for each article submitted. The Bulletin requires the last three items; that is, all but the abstract.

The Council confirmed the introduction of a new form of abstract blank for use in the presentation of ten-minute contributed papers. 
The principal new feature is the opportunity and requirement for the author to classify his abstract in one of sixty-six major categories corresponding to the major categories of Mathematical Reviews and the Mathematical Offprint Service. The advantage to the author and to the readers of the NOTICES is the grouping of abstracts in the printing according to the author's classification. Although the Associate Secretaries may find the classification useful, they cannot promise to follow it in grouping papers on the program for oral presentation and they are not expected to do so.

The Council accepted the report of the Nominating Committee for the Election of October 1969. The Committee presented the following candidates:

President Elect:

Vice-President (two to be elected):

Associate Secretaries:

Bulletin Editorial Committee:

Proceedings Editorial Committee:

Colloquium Editorial Committee:

Mathematical Reviews Editorial Committee:

Mathematical Surveys Editorial Committee:

Transactions and Memoirs Editorial

Committee:

Member-at-Large of the Council

(five to be elected):
N. Jacobson

A. P. Calderon

E. E. Floyd

P. Lax

I. M. Singer

P. T. Bateman

R. S. Pierce

M. H. Protter

George Seligman

Joseph Rotman

S. Mac Lane

Oscar Goldman

B. Yood

S. Sternberg

S. Armentrout

R. Steinberg

M. F. Atiyah

P. Hartman

Edward Nelson

M. Artin

F. B. Wright

H. B. Keller

R. K. Lashof

C. C. Moore

C. C. Lin

G. W. Mackey

Trustee:

The Council approved the nomination of H. P. McKean, Jr. and 
S. Lang as representatives of the Society in the Division of Mathematical Sciences of the National Research Council-National Academy of Science.

The tellers of the Referendum of May 1969 announced the following results:

$\begin{array}{lcc} & \text { FOR } & \text { AGAINST } \\ \text { Resolution B } & 6731 & 538 \\ \text { Resolution 1 } & 1860 & 5308 \\ \text { Resolution 2 } & 1903 & 5181 \\ \text { Resolution 3 } & 1167 & 5980 \\ \text { Resolution 4 } & 1998 & 5171 \\ \text { Resolution 5 } & 1884 & 5243\end{array}$

The text of Resolutions 1 through 5 may be found in Vol. 75, p. 480 , of this Bulletin. The text of Resolution B may be found on p. 737 of the same volume. The Council accepted the report of the Tellers, noting that the vote was FOR Resolution B and AGAINST Resolutions 1 through 5.

The Council approved the following resolution: The President is authorized to appoint a committee to investigate what opportunities to enter mathematics as a study or a career are available or are denied to various disadvantaged groups.

The Council has approved an amendment to the By-laws. If adopted by the membership, it has the following effect. The Committee to Monitor Problems in Communication, formerly appointive, is to be elected by the membership. The Committee elects its own Chairman who is then a member of the Council with one full vote.

At the Business Meeting of January 1969, an amendent to the By-laws was proposed. Article 10, Section 1, of the By-laws, describing the powers of a Business Meeting reads as follows:

A business meeting of the Society shall take final action only on business accepted by unanimous consent, or business notified to the full membership of the Society in the call for the meeting.

The proposal was to add certain words. The Council amended the proposal and approved it in amended form for submission to the membership. The added words are as follows:

... except that the business meetings held at either the Annual Meeting or at the Summer Meeting may take final action on business which has been recommended for consideration by the Council and has been accepted by a vote of four-fifths of the members of the Society present and voting at such a meeting.

The Council passed the following resolution unanimously: 
Whereas Edward L. Dubinsky, a tenured Associate Professor of Mathematics at Tulane University, is being dismissed after a faculty committee investigated charges against him at the request of the President and recommended Professor Dubinsky's retention, but with reprimand and warning; Therefore be it resolved that the Council of the American Mathematical Society in Eugene, Oregon on 26 August 1969 deplores the decision of the Board of Administrators of Tulane University to go against the advice of its faculty committee and urges reconsideration of this action, and further be it resolved that the Secretary send by telegram a copy of this resolution to the President and Board of Administrators of Tulane University.

The Council adjourned at 5:50 P.M. on August 27, 1969.

The Business Meeting convened at 4:00 P.M. on August 28, 1969 in the Ballroom of the Erb Memorial Union. Ex-president Charles B. Morrey, Jr. was in the chair. He appointed Professor Melvin Henriksen to be parliamentarian.

The Secretary reported actions of the Council of April 4, 1969, and August 26-27, 1969.

The Business Meeting passed the following motion:

Be it resolved that this Business Meeting of the American Mathematical Society supports the Council's resolution concerning the dismissal of Professor Ed Dubinsky from Tulane.

The Business Meeting passed the following resolution.

That this meeting urge the Society's President to be certain that there is full representation on this committee [to investigate what opportunities to enter mathematics as a study or a career are available or are denied to various disadvantaged groups] from among mathematicians from disadvantaged groups who are directly affected by the problems to be studied.

Other resolutions were introduced and discussed but no definitive action was taken. The meeting adjourned at 6:00 P.M., effectively ending all unfinished business.

Bethlehem, Pennsylvania

Everett Pitcher Secretary

R. S. PIERCE

Seattle, Washington Associate Secreatry 